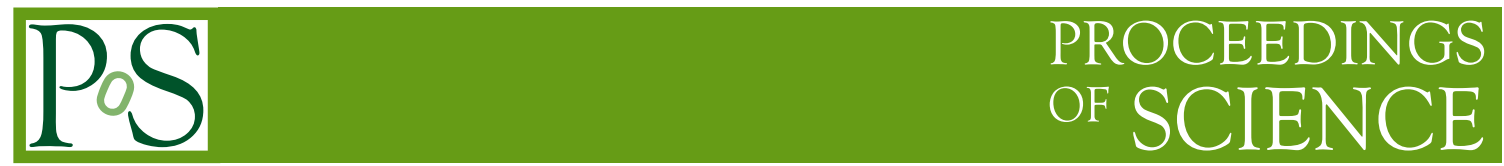

\title{
High Mass neutral / MSSM Higgs searches from CMS
}

\section{Adinda de Wit* on behalf of the CMS collaboration}

Imperial College Sci., Tech. \& Med. (UK)

E-mail: adinda.maite.de.witecern.ch

Searches for heavy neutral Higgs bosons and heavy resonances decaying to a $125 \mathrm{GeV}$ Higgs boson using the CMS detector are presented. This includes searches in different final states such as $\mathrm{W}$ and Z-bosons, bottom quarks, and tau leptons.

Fourth Annual Large Hadron Collider Physics

13-18 June 2016

Lund, Sweden

${ }^{*}$ Speaker. 


\section{Introduction}

The discovery of a standard model-compatible Higgs boson with a mass of $125 \mathrm{GeV}$ by the ATLAS and CMS collaborations in 2012 [1, 2], has impacted searches for physics beyond the standard model (BSM). BSM theories now need to allow for the $125 \mathrm{GeV}$ Higgs boson and its couplings as measured at the LHC. Many BSM theories predict the presence of 2 Higgs doublets instead of the one predicted by the standard model, leading to 5 Higgs bosons of which three are neutral: two scalars and one pseudoscalar. The generic class of two Higgs doublet models (2HDMs) [3, 4, 5] have the masses of the Higgs bosons, the ratio of the vacuum expectation values of the two Higgs doublets $(\tan \beta)$ and the mixing angle between the Higgs doublets $(\alpha)$ as free parameters. Different types of 2HDMs exist, where the type determines how the free parameters describe the couplings of the Higgs bosons to other particles. The minimally supersymmetric standard model (MSSM) $[6,7]$ forms a subset of type II 2HDMs motivated by SUSY and, at tree level, has two free parameters usually taken as the mass of the pseudoscalar $\mathrm{A}$ boson and $\tan \beta$, with additional parameters entering through radiative corrections fixed in different benchmark scenarios [8]. Other theories, such as models with warped extra dimensions $[9,10,11]$, predict heavy resonances that can decay to a pair of $125 \mathrm{GeV}$ Higgs bosons. In some versions of 2HDMs or the MSSM, heavier Higgs bosons can also decay into a pair of $125 \mathrm{GeV}$ Higgs bosons.

In these proceedings a collection of different results is presented. First, a summary of searches for BSM Higgs bosons using the data collected during Run I of the LHC, interpreted in the context of the MSSM, is presented (section 2). In addition, searches performed with data collected at $\sqrt{s}=13 \mathrm{TeV}$ during the $2015 \mathrm{LHC}$ run, corresponding to an integrated luminosity of $2.3-2.7$ $\mathrm{fb}^{-} 1$, are discussed. This includes searches for a heavy resonance decaying to a pair of $125 \mathrm{GeV}$ Higgs bosons (section 3), in the final states of $b \bar{b} b \bar{b}$ (section 3.1), $b \bar{b} W W$ (section 3.2) and $b \bar{b} \tau \tau$ (section 3.3), plus searches for heavy Higgs bosons directly decaying to a pair of $Z$ bosons (section 4) and for heavy Higgs bosons decaying to a pair of $\tau$ leptons (section 5).

\section{Run I BSM Higgs summary results}

During Run I of the LHC, searches for heavy Higgs bosons were performed in many channels. Figure 1 shows the observed and expected exclusion at 95\% confidence level from analyses in different final states, in the $m_{h}^{\bmod +}$ and hMSSM scenarios, two MSSM benchmark scenarios which allow for a $125 \mathrm{GeV}$ Higgs boson over a large part of the $m_{A}, \tan \beta$ plane. In the $m_{h}^{\bmod +}$ scenario, the whole $\tan \beta$ range is excluded below $m_{A}$ of $120 \mathrm{GeV}$ by a search for $H^{ \pm} \rightarrow \tau \nu$. For higher masses, a large part of the high $\tan \beta$ region is excluded by $H \rightarrow \tau \tau$ searches, with additional exclusion power in this region by searches for $H \rightarrow b \bar{b}$ and $H \rightarrow \mu \mu$. Exclusion in the low $\tan \beta$ region below masses of around $200 \mathrm{GeV}$ is closed off by $H \rightarrow W W / Z Z$ searches, though this region of the model is already not compatible with a $125 \mathrm{GeV}$ Higgs boson within the theoretical uncertainty of $3 \mathrm{GeV}$. The hMSSM scenario is excluded below $m_{A}=300 \mathrm{GeV}$ by the couplings of the $125 \mathrm{GeV}$ Higgs boson. The $H \rightarrow \tau \tau, H \rightarrow b \bar{b}, H \rightarrow \mu \mu, H \rightarrow W W / Z Z$ searches exclude similar regions in this model as in the $m_{h}^{\bmod +}$ scenario. In addition there is some exclusion power from $H \rightarrow h h$ searches at low $\tan \beta$. 
Figure 1 shows that a large part of the parameter space in MSSM benchmark scenarios was already ruled out by searches in Run I, but at higher masses and intermediate $\tan \beta$ there is still room for the MSSM, which means these analyses are still an important part of the physics programme in Run II.

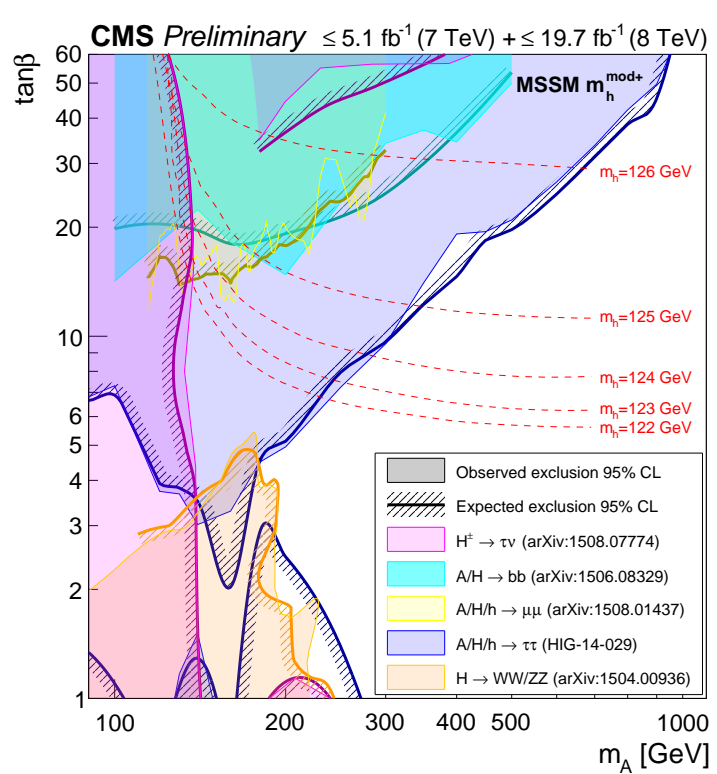

(a) $m_{h}^{\bmod +}$-scenario

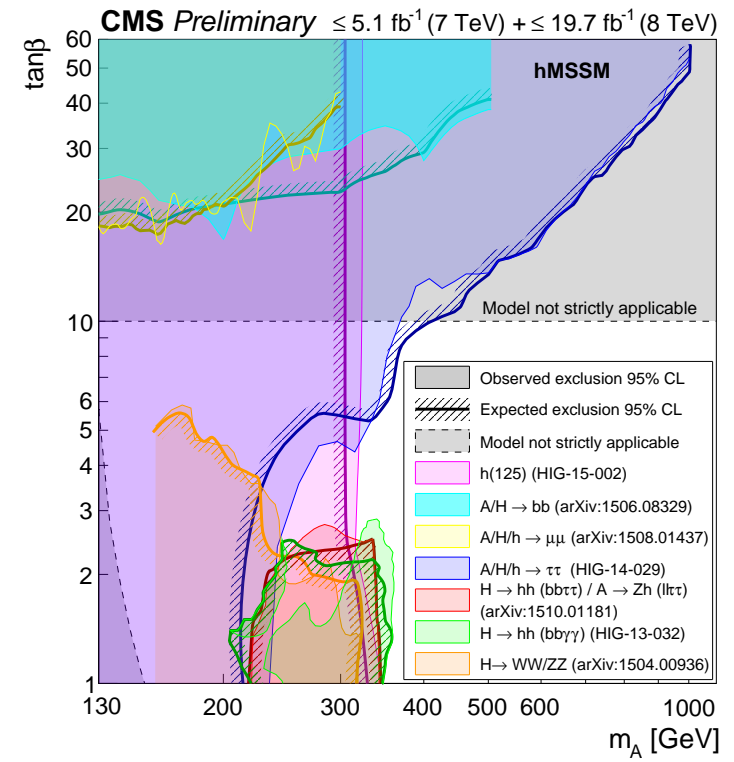

(b) hMSSM scenario

Figure 1: Exclusion limits of searches performed by the CMS experiment during Run I of the LHC, in the $m_{h}^{\bmod +}$ - (left) and hMSSM (right) scenarios of the MSSM. The shaded regions correspond to the observed exclusion from different analyses, the solid lines to the expected exclusion [12].

\section{Searches for $X \rightarrow h h$}

Three searches for a heavy resonance decaying to a pair of Higgs bosons are discussed in this section. The chosen final states of $b \bar{b} b \bar{b}$ (sect 3.1), $b \bar{b} W W$ (section 3.2) and $b \bar{b} \tau \tau$ (section 3.3) are chosen for their relatively large branching ratios, in combination with the cleaner $\tau \tau$ and $W W$ final states.

The common strategy for $H \rightarrow h h$ searches is to use the information of the $125 \mathrm{GeV}$ Higgs boson by selecting $\mathrm{h}$ candidates with an invariant mass compatible with $125 \mathrm{GeV}$.

\section{1 $X \rightarrow h h \rightarrow b \bar{b} b \bar{b}$}

The search for a heavy resonance $\mathrm{X}$ decaying to $h h$ with a final state of $4 \mathrm{~b}$ quarks [13] is a search for a narrow width resonance with spin 0 or spin 2, in a mass range of $260-1200 \mathrm{GeV}$. The analysis is performed on $2.3 \mathrm{fb}^{-1}$ of data collected at $\sqrt{s}=13 \mathrm{TeV}$. To reconstruct the diHiggs candidate, events with four b-tagged jets are selected. To maximise the expected sensitivity the search region is split into a low-mass region (260-400 GeV) and a medium mass region (400$1200 \mathrm{GeV}$ ). In the low mass region di-Higgs candidates are chosen such that the invariant mass of 
both Higgs candidates is close to $125 \mathrm{GeV}$. In the medium mass region the candidates are chosen such that the $\Delta R=\sqrt{\Delta \phi^{2}+\Delta \eta^{2}}$ between jets is small. The resonance mass $m_{X}$, used for signal extraction, is determined using a kinematic fit - the shape of the dominant QCD multijet background is estimated by fitting the $m_{X}$ distribution in a sideband in data.

The results of this analysis are model-independent upper limits on $\sigma \times \mathrm{BR}$, shown in figure 2 . Theoretical cross sections for a radion are overlaid on the upper limits for the spin-0 hypothesis (left), with theoretical cross-sections for a Kaluza Klein Graviton overlaid on the spin-2 upper limits (right). The radion is excluded with masses above $350 \mathrm{GeV}$, the Kaluza-Klein graviton with masses between 350 and $650 \mathrm{GeV}$.

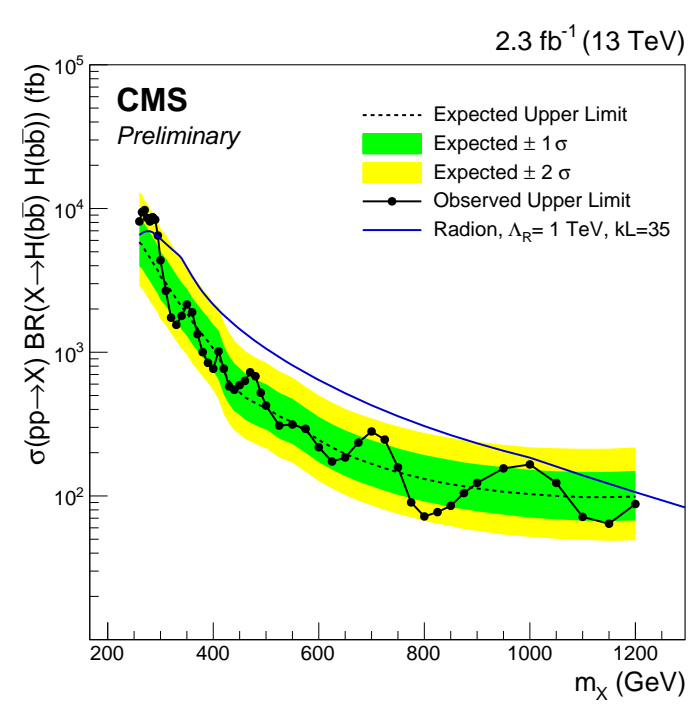

(a) Spin 0

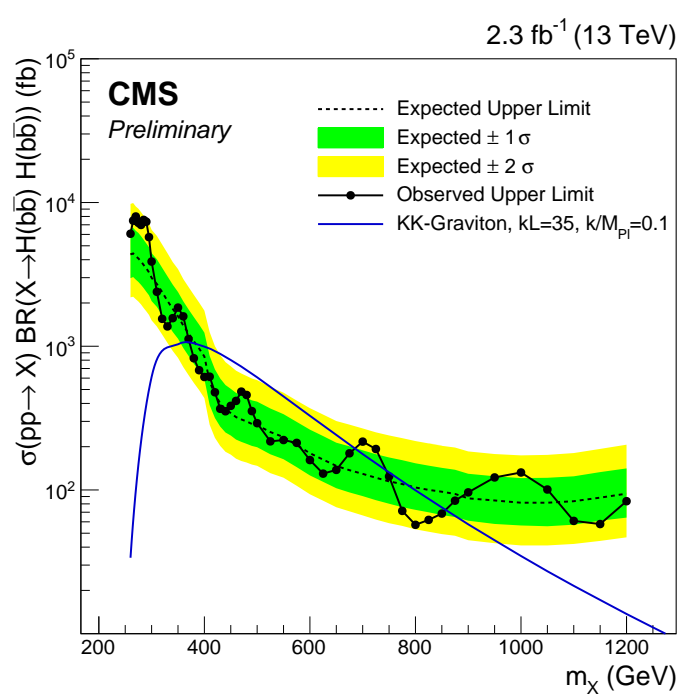

(b) Spin 2

Figure 2: Model-independent upper limits on $\sigma \times$ BR for the $X \rightarrow h h \rightarrow b \bar{b} b \bar{b}$ analysis, for the spin 0 (left) and spin 2 (right) hypotheses. Theoretical cross-sections for a radion (Kaluza-Klein graviton) are overlaid on the spin-0 (spin-2) hypothesis limits [13].

\section{2 $X \rightarrow h h \rightarrow b \bar{b} W(l v) W(l v)$}

The search for a heavy resonance $\mathrm{X}$ decaying to $h h$ with a final state of $2 \mathrm{~b}$ quarks and to $W$ bosons, which decay on to a lepton and a neutrino [14], is a search for a spin-0 (radion or typeI/II 2HDM) or a spin 2 (Kaluza-Klein Graviton) resonance, in a mass range of 260-900 GeV. To select events in the final state considered, events with two b-tagged jets and two oppositely charged leptons (electron or muon) are chosen. To separate signal from the dominant $t \bar{t}$ background, BDTs are trained on kinematic variables. In the mass range $260-450 \mathrm{GeV}$ a BDT trained on the signal sample at $400 \mathrm{GeV}$ is used, in the $450-900 \mathrm{GeV}$ mass range a BDT trained on the $650 \mathrm{GeV}$ signal sample is used. The BDT output distribution for backgrounds and different signal samples, using the high-mass range BDT, is shown in figure 3. 


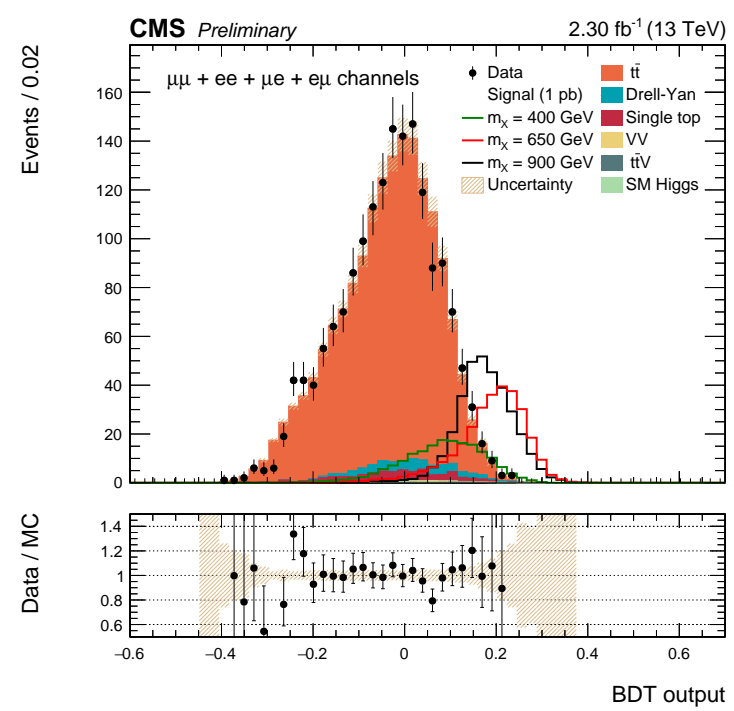

Figure 3: BDT output of BDT used to separate signal from the dominant $t \bar{t}$ background, in the high mass range [14].

For each BDT 4 categories are defined by splitting into low- and high BDT scores, and by considering two di-jet mass windows ( $95 \mathrm{GeV}<m_{j j}<135 \mathrm{GeV}$ and $m_{j j}$ outside of this window) . Using these four categories, upper limits on $\sigma \times \mathrm{BR}$ are set, as shown in figure 4

3.3 $X \rightarrow h h \rightarrow b \bar{b} \tau \tau$

The search for a heavy resonance $\mathrm{X}$ decaying to $h h$ with a final state of $2 \mathrm{~b}$-quarks and two $\tau$ leptons [15] is performed in a mass range of $260-900 \mathrm{GeV}$. Events are selected by requiring two b-tagged jets and a di- $\tau$ pair with both taus decaying hadronically, or one tau decaying hadronically and one decaying to an electron or a muon. To reject some fo the backgrounds while retaining most of the signal, a the di- $\tau$ and di-b invariant mass are both required to lie between 80 and $160 \mathrm{GeV}$. The mass of the di-Higgs candidate pair is reconstructed using a kinematic fit which assumes the mass of the Higgs boson is $125 \mathrm{GeV}$. Figure 5 (left) shows the kinematic fit mass distribution in the $b \bar{b} \mu \tau_{h}$ final state, with $\tau_{h}$ a hadronically decaying $\tau$. By fitting the kinematic fit mass distribution in the three channels simultaneously, upper limits on $\sigma \times \mathrm{BR}$ are obtained. These limits are shown in the right-hand side panel of figure 5.

\section{4. $H \rightarrow Z Z$}

This comprises a search for $H \rightarrow Z Z \rightarrow 2 l 2 v$ [16] and a search for $H \rightarrow Z Z \rightarrow 4 l$ [17]. In the $2 l 2 v$ final state events are selected by requiring a $Z \rightarrow l l$ candidate and large missing transverse energy. To maximise the sensitivity of the search, events are divided into three categories: a VBF category, a category with at least one jet and a 0 -jet category. The transverse mass of the di-lepton candidate and the missing transverse energy is used for signal extraction. The analysis is interpreted in, for example, a type II 2HDM. This is shown in figure 6 


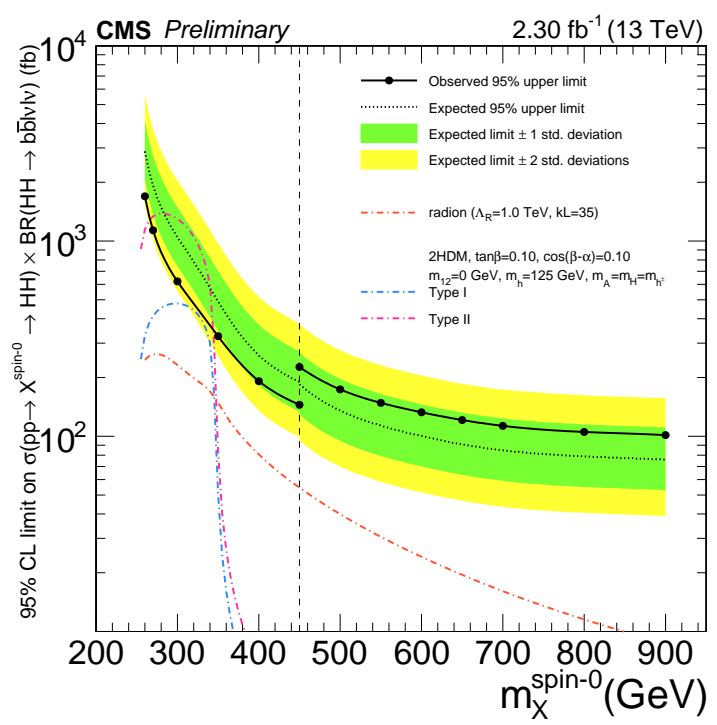

(a) Spin 0

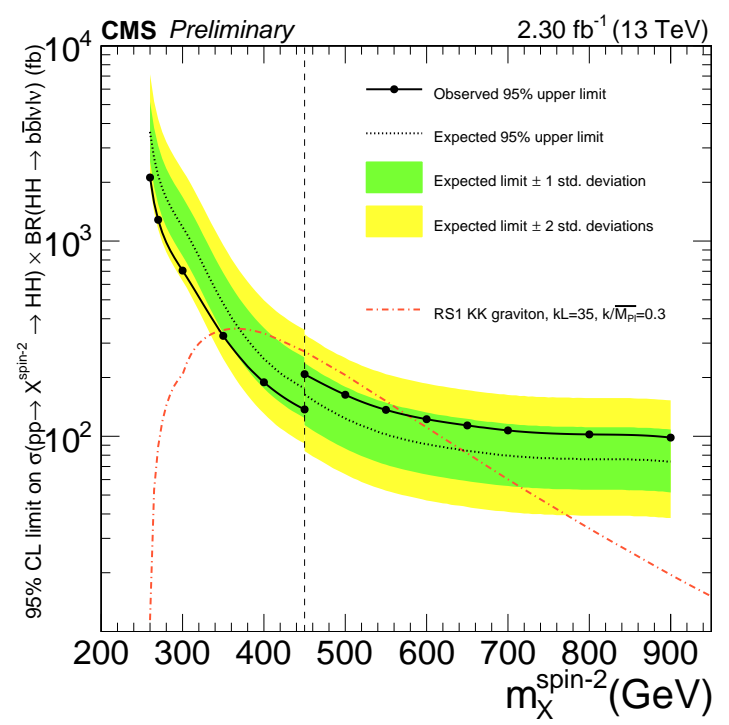

(b) Spin 2

Figure 4: Model-independent upper limits on $\sigma \times$ BR for the $X \rightarrow h h \rightarrow b \bar{b} W W$ analysis, for the spin 0 (left) and spin 2 (right) hypotheses. Theoretical cross-sections for a radion and examples of a type I and II $2 \mathrm{HDM}$ are overlaid on the spin 0 upper limit. The theoretical cross-section for a Kaluza-Klein graviton is overlaid on the spin-2 limits [14].

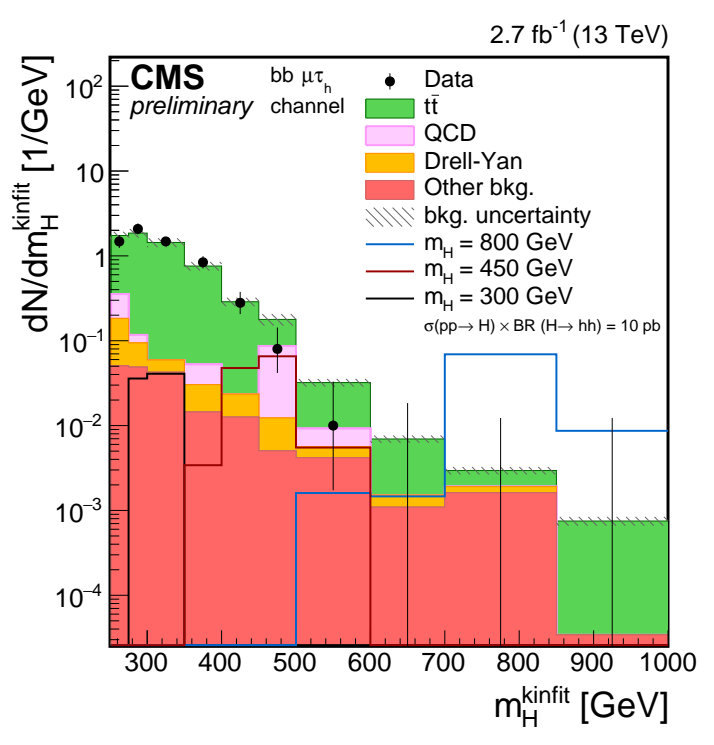

(a) Kinematic fit mass

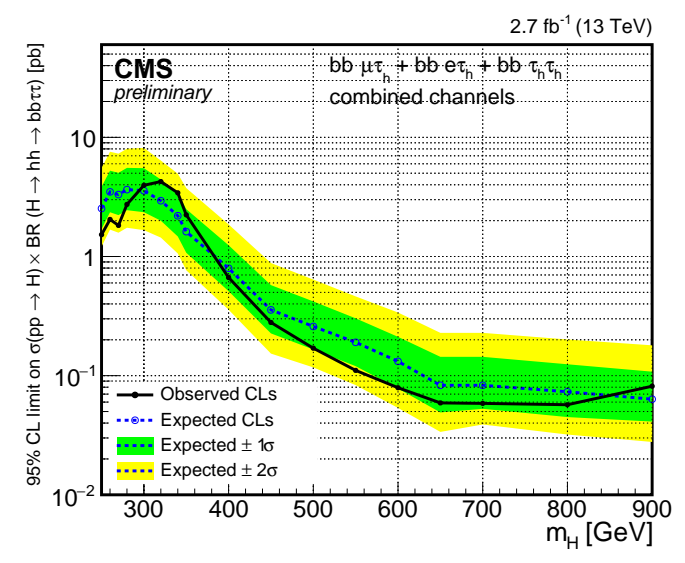

(b) Upper limits

Figure 5: Kinematic fit mass distribution of observed events, backgrounds, and signal at different masses, in the $b \bar{b} \mu \tau_{h}$ final state (left), and the model-independent upper limit on $\sigma \times \mathrm{BR}$ obtained by fitting the kinematic fit mass distributions in the three channels simultaneously (right) [15]. 


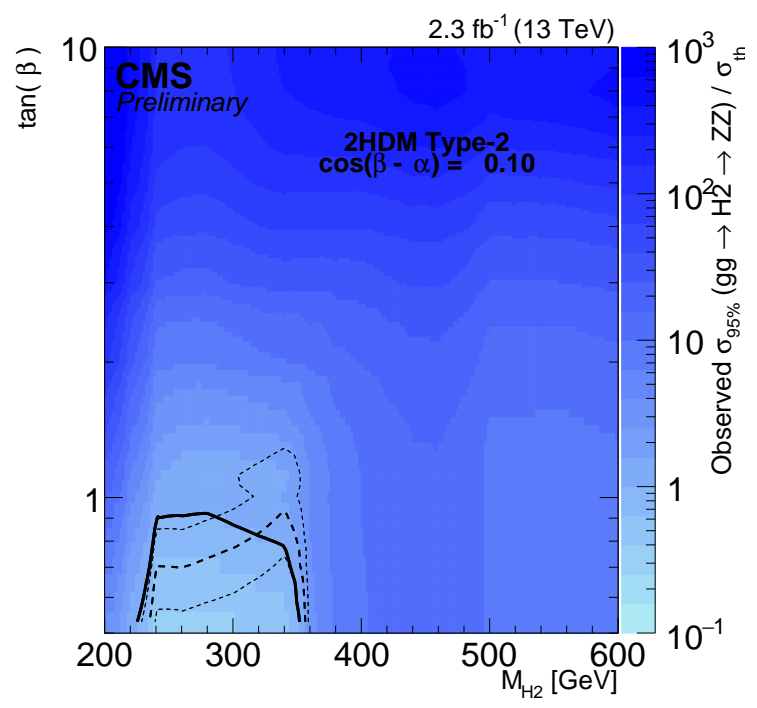

Figure 6: Observed and expected exclusion obtained by the search for $H \rightarrow Z Z \rightarrow 2 l 2 v$ in the $M_{H}, \tan \beta$ plane of an example of a type-II $2 \mathrm{HDM}$. A region between $220-360 \mathrm{GeV}$ is excluded below around $\tan \beta=0.8$ in this choice of 2HDM [16].

In the $4 l$ final state events are selected by requiring two $Z \rightarrow l l$ candidates. The 4-lepton invariant mass is used for signal extraction. This search sets upper limits on $\sigma \times \mathrm{BR}$ in a mass range of $150-1000 \mathrm{GeV}$ for resonances with widths of $0,5,20$, and $40 \mathrm{GeV}$, as shown in the left-hand side panel of figure 7. In addition, this search is interpreted in an example of a type-II 2HDM as shown in the right-hand side panel of figure 7.

\section{5. $H / A \rightarrow \tau \tau$}

The search for $H / A \rightarrow \tau \tau$ [18] is performed in a mass range from $80 \mathrm{GeV}$ up to $3.2 \mathrm{TeV}$ in 4 final states: both taus decaying hadronically, one of the taus decaying hadronically and the other decaying to an electron or muon, or one tau decaying into an electron and one decaying to a muon. This analysis was performed with a focus on the MSSM, where at high $\tan \beta$ the branching ratio of heavy Higgs bosons into a pair of $\tau$ leptons can be enhanced. In this regime the production of a Higgs boson in association with two b-jets also has a sizeable cross-section, so to target both this production mode and the production of a Higgs boson through gluon-gluon fusion, two categories are defined for each final state: the no b-tag category, which requires exactly 0 b-tagged jets, and the b-tag category, requiring at least $1 \mathrm{~b}$-tagged jet. The reconstructed di- $\tau$ transverse mass is fit in all channels and categories to set model-independent upper limits on $\sigma \times B R$. The distribution of the di- $\tau$ transverse mass in the $\mu \tau_{h}$ channel can be found in figure 8 , for the no b-tag category on the left, and the b-tag category on the right.

The 95\% CL upper limits on $\sigma \times \mathrm{BR}$ for the combination of all channels and categories is shown in figure 9, for the gluon-gluon fusion production process on the left and for $b \bar{b}$ associated production on the right. 


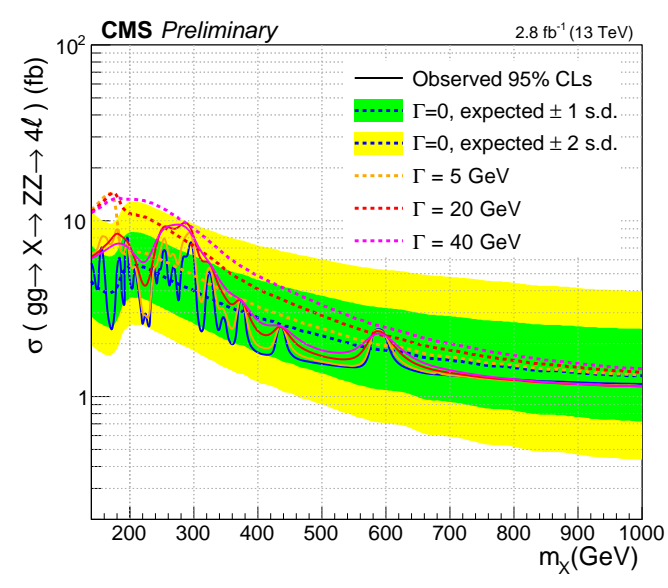

(a) Upper limits

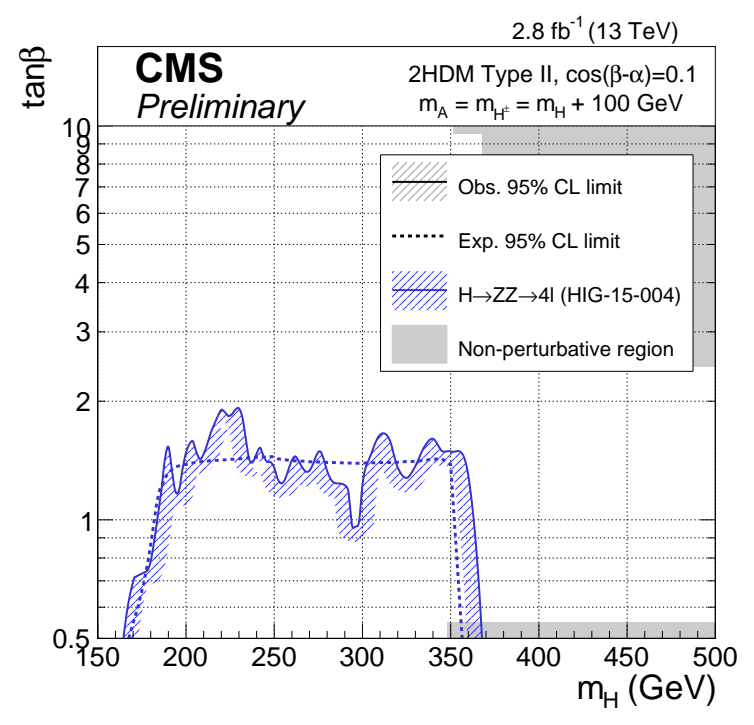

(b) Interpretation in type-II $2 \mathrm{HDM}$

Figure 7: $95 \% \mathrm{CL}$ upper limits on $\sigma \times \mathrm{BR}$ for the $H \rightarrow Z Z \rightarrow 4 l$ analysis (left) [17]. The different coloured lines correspond to different resonance widths. The right-hand side panel of this figure shows the interpretation of this search in an example of a type-II 2HDM, where a region between $160-360 \mathrm{GeV}$ is excluded below $\tan \beta$ around 1.2-2 [17].

As the analysis was carried out with an MSSM focus, model-dependent exclusion limits were set in for example $m_{A}-\tan \beta$ plane of the $m_{h}^{\bmod +}$ scenario. The MSSM signal hypothesis was tested against a standard model Higgs (at $125 \mathrm{GeV}$ ) + background hypothesis. The resulting exclusion limits are shown in figure 10. Comparing the observed and expected exclusion limits from this analysis with those obtained by the most sensitive $7+8 \mathrm{TeV}$ analysis (blue lines in figure 10) one can see that the $13 \mathrm{TeV}$ analysis observes a larger exclusion from around $m_{A}=300 \mathrm{GeV}$, and that the analysis surpasses the expected sensitivity from Run I starting from $m_{A}=600 \mathrm{GeV}$, despite the much smaller dataset. 


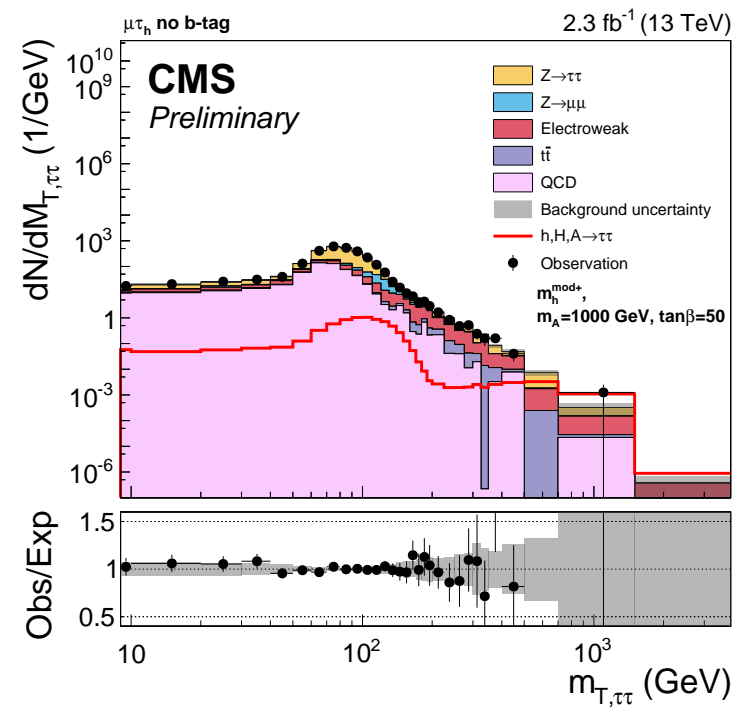

(a) no b-tag

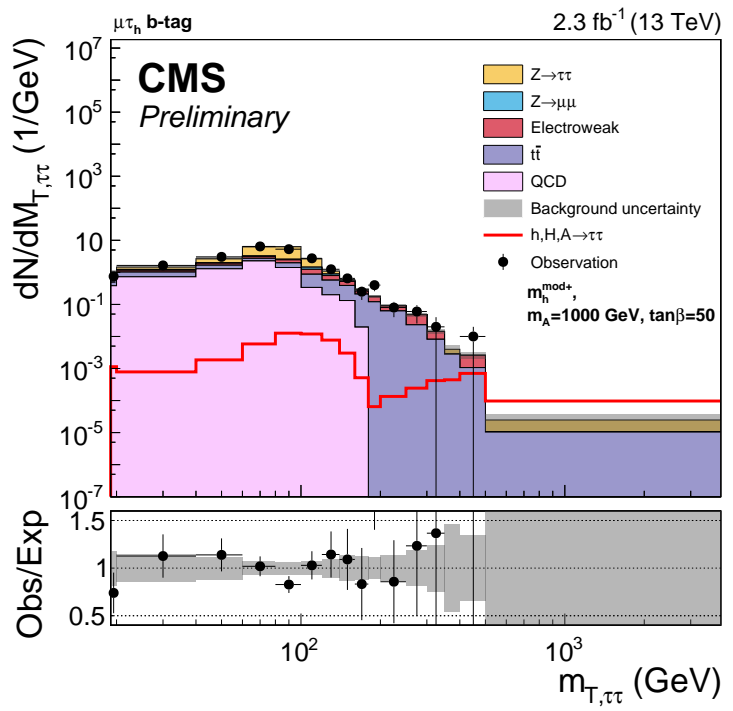

(b) b-tag

Figure 8: Reconstructed di- $\tau$ transverse mass distribution in the no b-tag (left) and b-tag (right) categories of the $\mu \tau_{h}$ channel. The red line is the combined signal template for the three neutral Higgs bosons, at a benchmark point of $m_{A}=1 \mathrm{TeV}$ and $\tan \beta=50$ in the $m_{h}^{\bmod +}$ scenario [18].

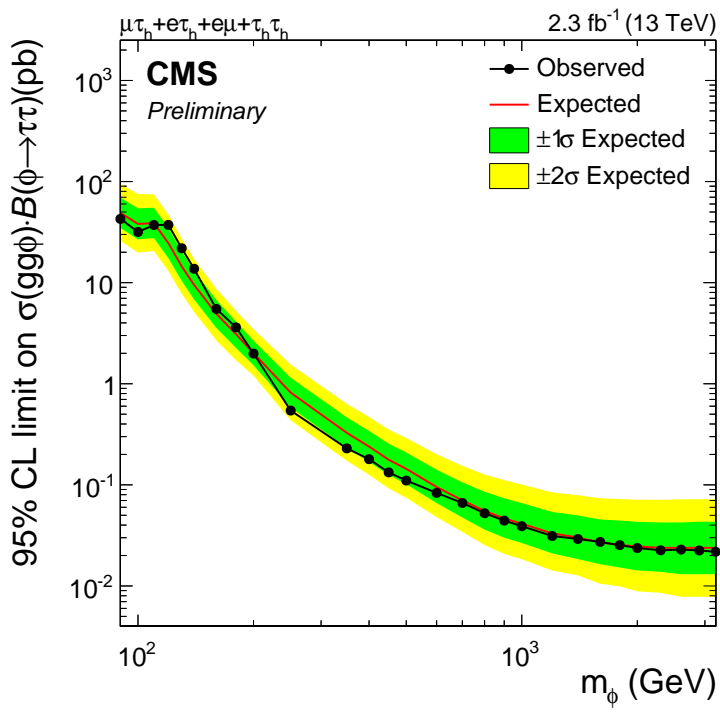

(a) gluon-gluon fusion production

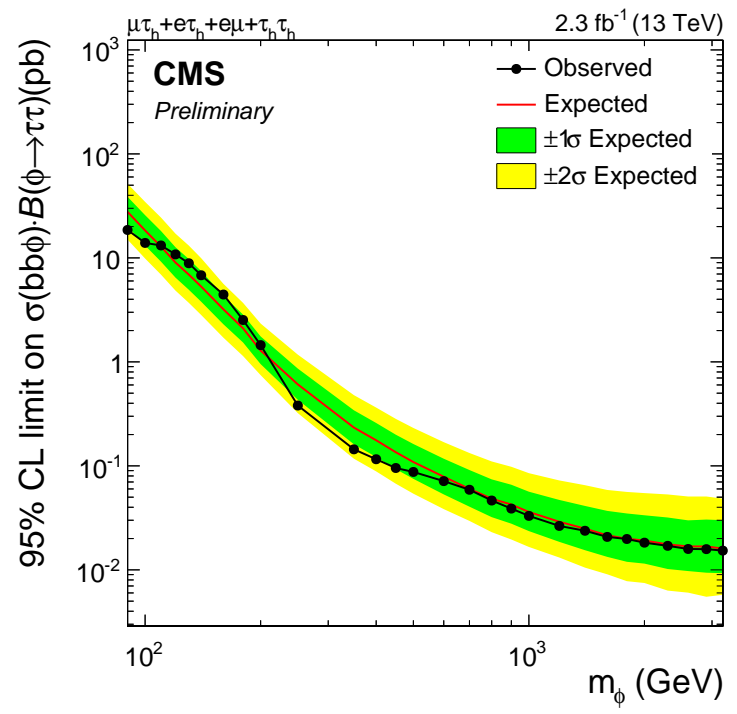

(b) $b \bar{b}$ associated production

Figure 9: Upper limits on $\sigma \times$ BR for the gluon-gluon fusion production process (left) and $b \bar{b}$ associated production (right) [18]. 


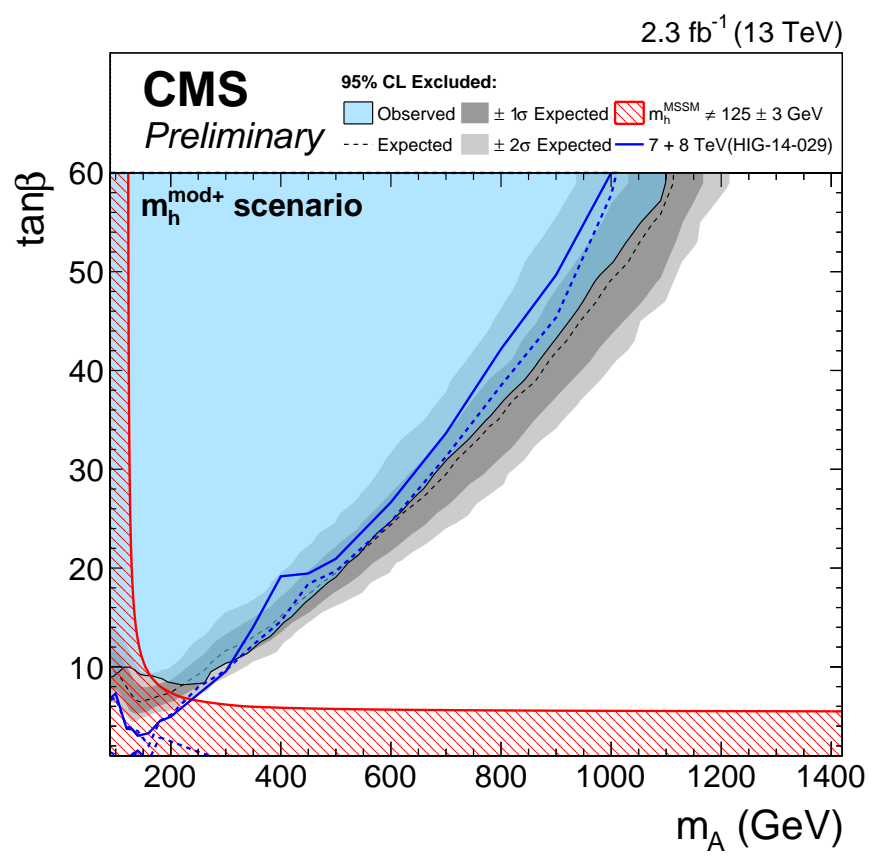

Figure 10: Exclusion limits in the $m_{A}-\tan \beta$ plane of the $m_{h}^{\bmod +}$ scenario. The blue shaded region indicates the observed exclusion, the dashed black line the expected exclusion. Overlaid on this plot are the observed (solid blue) and expected (dashed blue) limits obtained by the most sensitive $7+8 \mathrm{TeV} H \rightarrow \tau \tau$ analysis. The red shaded area is excluded by the requirement of the presence of a Higgs boson at $125 \mathrm{GeV}$ [18].

\section{References}

[1] ATLAS Collaboration, "Observation of a new particle in the search for the Standard Model Higgs boson with the ATLAS detector at the LHC", Phys. Lett. B 716 (2012) doi :tt10.1016/j.physletb.2012.08.020, arXiv:1207. 7214.

[2] CMS Collabotation, "Observation of a new boson at a mass of $125 \mathrm{GeV}$ with the CMS experiment at the LHC", Phys. Lett. B716 (2012), do $\mathrm{i}: \mathrm{tt10.1016/j.physletb.2012.08.021,}$ arXiv: 1207.7235.

[3] S.L. Glashow and S. Weinberg, "Natural conservation laws for neutral currents", Phys. Rev.D 15 (1977) 1958, doi : tt10.1103/PhysRevD.151958.

[4] J.F. Gunion, H.E. Haber, G.L. Kane et. al. "The Higgs Hunter's Guide", Front. Phys. 80 (2000) 1.

[5] G. Branco, P. Ferretira, L. Lavoura et. al. "Theory and phenomenology of two-Higgs-doublet models", Phys. Rept.516 (2012) 1, doi:tt10.1016/j.physrep.2012.02002, arXiv:1106.0034.

[6] P. Fayet, "Supergauge invariant extension of the Higgs mechanism and a model for the electron and its neutrino", Nucl. Phys. B90 (1975) 104, doi : 10.1016/0550-3213(75) 90636-7. 
[7] P. Fayet, "Spontaneously broken supersymmetric theories of weak, electromagnetic and strong interactions", Phys. Lett. B69 (1977) 489, do i : 10.1016/0370-2693(77)90852-8.

[8] P. Bechtle et al., "Probing the Standard Model with Higgs signal rates from the Tevatron, the LHC and a future ILC", JHEP 11 (2014) 039, do i : 10.1007/JHEP11(2014)039, arXiv: 1403.1582 .

[9] W.D. Goldberger and M.S. Wise, "Modulus stabilization with bulk fields", Phys.Rev.Lett. 83 (1999) 33703373.

[10] C. Csaki, J. Hubisz, and S.J. Lee, "Radion phenomenology in realistic warped space Model", Phys. Rev. D 62 (2000) 045015.

[11] H.Davoudiasl, J.Hewett, and T. Rizzo, "Phenomenology of the Randall-Sundrum Gauge Hierarchy Model", Phys. Rev. Lett84 (2000) 2080.

[12] CMS Collaboration, "Summary of high mass BSM Higgs searches using CMS run-I data", CMS-PAS-HIG-16-007, CERN (2016).

[13] CMS Collaboration,"Search for resonant pair production of Higgs bosons decaying to two bottom quark-antiquark pairs in proton-proton collisions at $13 \mathrm{TeV}$ ", CMS-PAS-HIG-16-002, CERN (2016).

[14] CMS Collaboration, "Search for resonant Higgs boson pair production in the $b \bar{b} l v l v$ final state at $\sqrt{s}=13$ TeV", CMS-PAS-HIG-16-011, CERN (2016).

[15] CMS Collaboration, "Search for resonant Higgs boson pair production in the $b \bar{b} \tau^{+} \tau^{-}$final state", CMS-PAS-HIG-16-013, CERN (2016)

[16] CMS Collaboration, "Search for a heavy scalar boson decaying into a pair of $\mathrm{Z}$ bosons in the $2 l 2 v$ final state", CMS-PAS-HIG-16-001, CERN (2016)

[17] CMS Collaboration, "Studies of Higgs boson production in the four-lepton final state at $\sqrt{s}=13 \mathrm{TeV} "$, CMS-PAS-HIG-15-004, CERN (2016)

[18] CMS Collaboration, "Search for a neutral MSSM Higgs boson decaying into $\tau \tau$ at $13 \mathrm{TeV}$ ", CMS-PAS-HIG-16-006, CERN (2016). 